\title{
A correlation based fault detection method for short circuits in battery packs
}

\author{
Bing Xia ${ }^{\mathrm{a}, \mathrm{b}}$, Yunlong Shang ${ }^{\mathrm{a}}$, Truong Nguyen ${ }^{\mathrm{b}}$, and Chris $\mathrm{Mi}^{\mathrm{a}, *}$ \\ ${ }^{\mathrm{a}}$ Department of Electrical and Computer Engineering, San Diego State University, \\ 5500 Campanile Drive, San Diego, CA 92182, USA \\ ${ }^{\mathrm{b}}$ Department of Electrical and Computer Engineering, University of California San Diego, \\ 9500 Gilman Drive, La Jolla, CA 92093, USA \\ *Corresponding Author. Tel.: +1 (619) 594 3741; fax: +1 (619) 5942654 \\ Email address: bixia@eng.ucsd.edu (B. Xia), yshang@mail.sdsu.edu (Y. Shang), \\ tqn001@eng.ucsd.edu (T. Nguyen),cmi@sdsu.edu (C. Mi)
}

\begin{abstract}
This paper presents a fault detection method for short circuits based on the correlation coefficient of voltage curves. The proposed method utilizes the direct voltage measurements from the battery cells, and does not require any additional hardware or effort in modeling during fault detection. Moreover, the inherent mathematical properties of the correlation coefficient ensure the robustness of this method as the battery pack ages or is imbalanced in real applications. In order to apply this method online, the recursive moving window correlation coefficient calculation is adopted to maintain the detection sensitivity to faults during operation. An additive square wave is designed to prevent false positive detections when the batteries are at rest. The fault isolation can be achieved by identifying the overlapped cell in the correlation coefficients with fault flags. Simulation and experimental results validated the feasibility and demonstrated the advantages of this method.
\end{abstract}

Keywords: lithium-ion batteries; fault diagnosis; short circuit; correlation coefficient. 


\section{Introduction}

The prevailing application of lithium-ion batteries in the electrified vehicles (EVs) provides competitive energy and power performances [1]. However, many recent publications worldwide have reported a series of related accidents [2-4], revealing the potential safe concerns of lithiumion batteries, which retard their rapid expansion.

The demand of safe battery operation gives rise to the battery management systems (BMS) [5]. The BMS in an EV monitors the voltage, current and temperature of a battery pack, estimates the essential states of the cells $[6,7]$, and maintains the safe and efficient operation of the energy storage system $[8,9]$. A qualified BMS should be able to flag warnings when any of the cell is in abuse condition and activate the corresponding mitigation methods $[10,11]$. The abuse behaviors cover a wide variety of conditions which can be grouped into four categories based on the electric features, namely, over charge, over discharge, external short circuit and internal short circuit [12].

Compared with the over charge/discharge faults, the short circuit faults are more hazardous. The external short circuit fault induces abnormally high heat generation rate, which may easily initiate the thermal runaway. The thermal runway describes the heat propagation process within the battery pack, where the exothermic side reaction of one cell failure influences the safe operation of surrounding cells, and further triggers the chain exothermic reactions [13]. Thereafter, the heat builds up rapidly and finally results in fire or explosion. Different from the external short circuit, the internal short circuit is usually caused by manufacturing defects or mechanical failure. As the short area in the separator emerges, an internal current path is formed. The local high current rate elevates the local temperature. The high temperature first forces the 
closure of separators with shutdown function. However, if the heat is not dissipated well, an even higher temperature will melt the separator and eventually trigger thermal runaway [14].

With the available voltage, current and temperature measurements, previous research introduced many detection approaches specifically for short circuits, which can be classified into two groups: a) threshold based methods, and b) model based methods. The external short circuit fault can be detected by comparing the direct sensor measurements with the threshold in current increase, voltage drop and temperature rise $[15,16]$. The limitation of this method is that a fault may occur without triggering the threshold. If the resistance in the short loop is moderate such that the current, voltage and temperature does not go beyond the preset limit within a short period of time, the fault will not be flagged at the occurrence.

To a certain extent, the detection of the internal short circuit is more complicated. At the initial phase of internal short circuit fault, when the damage on the separator leads to an internal short, and the local heat results into the separator shutdown, the terminal voltage of the fault cell will first drop and then recover $[14,17,18]$. If a simple threshold based method is applied, it may not capture the abnormal voltage pattern. Since the current flows internally, it cannot be recorded by the current sensor either. To make it even worse, the fast short may not cause adequate increase of the cell surface temperature. Therefore, the threshold based methods do not function with satisfaction. An illustrative case has been studied in [2], where a voltage drop recovery was recorded by the onboard BMS, but the BMS failed to flag any warning at the initial phase of failure. When the fault was recognized 17 minutes later, the start of fire was inevitable.

An improved threshold based method sets the threshold for maximum voltage differences within a battery pack, instead of the absolute voltage value for each cell. This method was originally designed for imbalanced state detection [19], but can be adopted to short circuit 
detection as well. In this method, the threshold of voltage difference should be chosen with special care. If the value is too large, it cannot capture the voltage drop, whereas if the value is too small, it can easily lead to false positive detection due to inconsistencies in battery states and parameters.

To investigate the mechanism of the internal short circuits, extensive experiments were conducted to induce the faults by indentation [20-23], nail penetration [24, 25], fabrication with defect structures $[26,27]$ and extreme high temperatures $[28,29]$. Based on the findings, the models of the internal short circuits were built in $[30,31]$, which can be utilized to detect the fault by comparing the virtue model output/state with the battery output/state. If the residue is above a threshold, a fault is flagged. A thorough introduction of multiple model based cell condition monitoring was presented in [32]. It was discussed in [33] that the changes in the estimated circuit parameters and temperature derivative of equilibrium potential are good indicators to identify internal short circuit faults. These methods give more reliable detection than the threshold based method, however, it requires substantial effort in accurate modeling and testing [34]. Except that, this method is still vulnerable to cell inconsistencies, unless the online parameter estimation is implemented for every single cell in the series pack which is prohibitive in real applications.

Other methods seek to improve the detection and isolation of internal short circuits from system design perspective. It was proposed in [35] that the internal short circuits can be identified by the differences of branch currents in the series-parallel battery pack, however, the method requires current sensors in every parallel branch, which greatly increases the system cost. The interleaved voltage measurement method was presented in [36], and can distinguish between 
sensor and cell failures without additional components, but the fault detection is still not robust to cell inconsistencies.

In order to avoid the influence of inconsistencies in the battery pack upon fault detection, this paper proposes a correlation based method for short circuit detection in lithium-ion battery packs. The main idea is to capture the unusual voltage variation at the initial phase of a short circuit fault by calculating the correlation coefficients of the cell voltages. Since the correlation coefficient is independent of the mean value and the amplitude of the fluctuations, when applying to the cell voltages, it eliminates the inconsistencies in open circuit voltage (OCV) and internal resistance, hence does not lead to false positive faults when the batteries are in different state of charge $(\mathrm{SoC})$ or state of health $(\mathrm{SoH})$. Moreover, this method is non-redundancy based, because the correlation coefficient calculation only involves voltage outputs from different cells, thereby saving the extensive effort in modeling and testing.

Based on these desired features, an online short circuit detection algorithm is developed. A moving window filter is utilized to forget past data and maintain the detection sensitivity to faults. An additive square wave is designed to prevent false detections when the noises dominate the voltage variations during rest periods. Simulations are used to illustrate the functions of the moving window and the square wave in different scenarios. Experiments are conducted to validate the feasibility of the method, and to demonstrate the fault isolation. Finally, the key assumptions of the proposed method are discussed and the root causes of different detection results from different methods are analyzed.

\section{Method description}

\subsection{A non-redundancy based fault detection method}


Generally, the existing fault diagnosis methods are redundancy based, which can be further divided into hardware redundancy and analytical redundancy. The key idea is to compare the system state/output with the state/output of redundancy systems, and flag a fault when the residue is not reasonably small. The hardware redundancy utilizes duplicative real systems to provide comparison. The common drawbacks of the hardware redundancy are its increase in hardware cost and system complexity, making it impractical for battery systems. The analytical redundancy constructs a mathematical system model and compares the model state/output with real state/output of the system. The aforementioned threshold based method can be regarded as a rough modeling of the system limit without considering the input. However, the battery system is highly nonlinear and a model is not assured to cover every situation. Then, the ambiguity emerges when a fault is indicated which may come from a true battery failure, or can be resulted from an inaccurate model. Other than that, extensive work is needed to build the system model and substantial effort is required in data acquisition and validation.

If a closer insight is given to the redundancy based fault diagnosis methods, it can be found that they are designed for single systems, or the target system is unique and can function individually without any duplicative parts. This is the reason why it requires either a hardware or analytical redundancy to provide a second output to compare with. Keep this in mind, it is worth noting that there is a fundamental difference in the battery system. A battery pack includes multiple same battery cells connecting in series, meaning that the cells share the same current. In other words, a battery system consists of multiple same systems with same inputs, and thus the voltage outputs should be similar, if not at fault conditions. In this way, one cell output can be compared with that of any other cells, or all the other cells can serve as the hardware redundancies of one single cell, even though there is physically no redundancy in the system. It 
is important to notice that this method is robust because the output comes from the real systems, which is guaranteed to be accurate and does not suffer from convergence issues.

\subsection{Correlation coefficient}

Rigorously speaking, the battery cells within a battery pack are not exactly the same. There are variations in the manufacturing process, thermal conditions in usage, balance state, etc. In general, all these variations are reflected into two essential states, i.e., SoC and SoH. These two states affect the static and dynamic behavior of a battery cell by different OCV and internal resistance, respectively. The different OCV leads to an offset in the cell voltages and the difference in internal resistance causes voltage fluctuations with different amplitudes. If the voltage outputs of the battery cells are simply compared, these voltage differences can easily exceed the preset threshold value, making this simple comparison not robust in real applications.

In statistics, correlation coefficient, or Pearson product-moment correlation coefficient, is a degree of measurement indicating the linear relation between two variables [37]. It is expressed as

$$
r_{X, Y}=\frac{\operatorname{cov}_{X, Y}}{\sigma_{X} \sigma_{Y}}=\frac{\sum_{i=1}^{n}\left(x_{i}-\mu_{x}\right)\left(y_{i}-\mu_{y}\right)}{\sqrt{\sum_{i=1}^{n}\left(x_{i}-\mu_{x}\right)^{2}} \sqrt{\sum_{i=1}^{n}\left(y_{i}-\mu_{y}\right)^{2}}}
$$

where $r_{X, Y}$ is the correlation coefficient of variables $X$ and $Y, \operatorname{cov}_{X, Y}$ is the covariance of $X$ and $Y$,

$\sigma_{Z}$ is the variance of variable $Z, \mu_{Z}$ is the mean value of variable $Z$, and $n$ is the number of samples in the data. The correlation coefficient is unitless, and ranges from +1 to -1 inclusive, where +1 indicates total positive correlation, 0 indicates no correlation and -1 indicates total negative correlation.

An important property of the correlation coefficient is given as 


$$
r_{\alpha X+\beta, Y}=r_{X, Y}
$$

where $\alpha$ and $\beta$ are two constants. This property is intuitive because when an offset $\beta$ is added to any of the variables, it is subtracted from the mean values in (1), and when the fluctuation amplitude of a variable is multiplied by $\alpha$, it multiplies both the numerator and denominator by $\alpha$. Hence, the correlation coefficient measures whether the trend of two curves matches, instead of their exact shape.

This feature is indeed an ideal property in coping with the inconsistencies in lithium-ion batteries when: a) the imbalanced batteries demonstrate different $\mathrm{OCV}$, and b) the cells in different aging levels exhibit different internal resistances. If the correlation coefficient of two cell voltages is calculated, the difference in OCV is removed because the static offset does not influence the correlation coefficient, and the difference in internal resistances is eliminated because the correlation coefficient is also independent of the fluctuation amplitudes. Therefore, ideally, the correlation coefficient of two series cell voltages should be close to +1 during normal operations. When a short circuit occurs, the abnormal voltage drop influences the synchronized fluctuation on battery voltages, thus being reflected by the reduced correlation coefficient.

\section{Extension to real applications}

\subsection{Recursive estimation}

For online implementation, the correlation coefficient should be calculate recursively. Eq. (1) is not a satisfactory formula for such application. Although the mean values can be updated after every sampling recursively, the subtractions from mean values have to be calculated individually.

An equivalent expression of the correlation coefficient can be obtained by multiplying both the numerator and denominator of (1) by $n$, as 


$$
r_{X, Y}=\frac{n \sum_{i=1}^{n} x_{i} y_{i}-\left(\sum_{i=1}^{n} x_{i}\right)\left(\sum_{i=1}^{n} y_{i}\right)}{\sqrt{n \sum_{i=1}^{n} x_{i}^{2}-\left(\sum_{i=1}^{n} x_{i}\right)^{2}} \sqrt{n \sum_{i=1}^{n} y_{i}^{2}-\left(\sum_{i=1}^{n} y_{i}\right)^{2}}}
$$

Eq. (3) does not require subtractions from the mean values, so it is more appropriate for recursive estimation. The formula of the recursive estimation is then obtained as

$$
\begin{aligned}
& P_{k}=P_{k-1}+x_{i} y_{i} \\
& Q_{k}=Q_{k-1}+x_{i} \\
& R_{k}=R_{k-1}+y_{i} \\
& S_{k}=S_{k-1}+x_{i}^{2} \\
& T_{k}=T_{k-1}+y_{i}^{2} \\
& \left(r_{X, Y}\right)_{k}=\frac{k P_{k}-Q_{k} R_{k}}{\sqrt{k S_{k}-Q_{k}^{2}} \sqrt{k T_{k}-R_{k}^{2}}}
\end{aligned}
$$

\subsection{Forgetting mechanism}

Eq. (4) can be used to obtain the similarity of the time domain trends for two voltage curves from the beginning of measurement. However, there are still several difficulties in implementation: a) if the fault occurs long time after the beginning of measurement, the abnormal behavior will have negligible effect on the correlation coefficient due to the high similarity of the long history data; b) as time goes by, the magnitudes of updated quantities in (4) become larger and larger, and will eventually exceed the storage limits of the onboard microprocessors.

The most straightforward approach to solving the abovementioned problems is to employ a moving window filter for data processing, i.e., at each time instant, the correlation coefficient for the data only in a history time window is calculated. Then (4) is modified as 


$$
\begin{aligned}
& P_{k}=P_{k-1}+x_{i} y_{i}-x_{i-w} y_{i-w} \\
& Q_{k}=Q_{k-1}+x_{i}-x_{i-w} \\
& R_{k}=R_{k-1}+y_{i}-y_{i-w} \\
& S_{k}=S_{k-1}+x_{i}^{2}-x_{i-w}^{2} \\
& T_{k}=T_{k-1}+y_{i}^{2}-y_{i-w}^{2} \\
& \left(r_{X, Y}\right)_{k}=\frac{w P_{k}-Q_{k} R_{k}}{\sqrt{w S_{k}-Q_{k}^{2}} \sqrt{w T_{k}-R_{k}^{2}}}
\end{aligned}
$$

where $w$ is the size of the moving window. It needs to be noted that (5) should be initialized by (4) in the first $w$ samples.

It is also worth noticing that the window size should be chosen with special care. If a large set of data is employed in the calculation, the abnormal voltage variation led by short circuit will have negligible effects in the correlation coefficient. Hence, in order to keep the detection sensitivity to faults, a moving window with a small size is preferred. On the other hand, when the moving window size is too small, the noise will be regarded as abnormal fluctuations and the measurement noises will influence the calculation as well. Therefore, a proper size of moving window should be selected based on the application.

\subsection{Special case when noises dominate}

When two signals are added to $X$ and $Y$, respectively, Eq. (1) can be derived as

$$
r_{X+N, Y+M}=\frac{\operatorname{cov}_{X+N, Y+M}}{\sigma_{X+N} \sigma_{Y+M}}=\frac{\sum_{i=1}^{n}\left(x_{i}-\mu_{x}+N_{i}-\mu_{N}\right)\left(y_{i}-\mu_{y}+M_{i}-\mu_{M}\right)}{\sqrt{\sum_{i=1}^{n}\left(x_{i}-\mu_{x}+N_{i}-\mu_{N}\right)^{2}} \sqrt{\sum_{i=1}^{n}\left(y_{i}-\mu_{y}+M_{i}-\mu_{M}\right)^{2}}}
$$

where $N$ is the signal added to $X$ and $M$ is the signal added to $Y$. Assume that both $N$ and $M$ are independent of $X$ and $Y$. Eq. (6) can be simplified as

$$
r_{X+N, Y+M}=\frac{\sum_{i=1}^{n}\left(x_{i}-\mu_{x}\right)\left(y_{i}-\mu_{y}\right)+\sum_{i=1}^{n}\left(N_{i}-\mu_{N}\right)\left(M_{i}-\mu_{M}\right)}{\sqrt{\sum_{i=1}^{n}\left(x_{i}-\mu_{x}\right)^{2}+\sum_{i=1}^{n}\left(N_{i}-\mu_{N}\right)^{2}} \sqrt{\sum_{i=1}^{n}\left(y_{i}-\mu_{y}\right)^{2}+\sum_{i=1}^{n}\left(M_{i}-\mu_{M}\right)^{2}}}
$$


There are two terms in the numerator of (7). When the batteries are at rest, the first term is zero because the voltages are very close to their OCVs. When $N$ and $M$ are independent and identically distributed white noises, the second term is zero as well. This indicates that the correlation coefficient is close to zero in this situation. This small value will lead to a sudden drop in the calculation and surely triggers a false positive fault, which is not desired.

If (7) is further expanded to three signals and preferably the mean values of the added signals are all zero, the correlation coefficient can be expressed as

$$
r_{X+A+N, Y+B+M}=\frac{\sum_{i=1}^{n}\left(x_{i}-\mu_{x}\right)\left(y_{i}-\mu_{y}\right)+\sum_{i=1}^{n} A_{i} B_{i}+\sum_{i=1}^{n} N_{i} M_{i}}{\sqrt{\sum_{i=1}^{n}\left(x_{i}-\mu_{x}\right)^{2}+\sum_{i=1}^{n} A_{i}^{2}+\sum_{i=1}^{n} N_{i}^{2}} \sqrt{\sum_{i=1}^{n}\left(y_{i}-\mu_{y}\right)^{2}+\sum_{i=1}^{n} B_{i}^{2}+\sum_{i=1}^{n} M_{i}^{2}}}
$$

where $A$ and $B$ are the newly added signals to $X$ and $Y$, and assume they are independent of $X, Y$, $N$ and $M$. A solution to avoiding the zero correlation coefficient is provided in (8) when A and B are dependent. In such cases, when the batteries are at rest, Eq. (8) is simplified as

$$
r_{X+A+N, Y+B+M}=\frac{\operatorname{cov}_{A, B}}{\sqrt{\sigma_{A}^{2}+\sigma_{N}^{2}} \sqrt{\sigma_{B}^{2}+\sigma_{M}^{2}}} \approx r_{A, B}, \sigma_{N}^{2}<<\sigma_{A}^{2}, \sigma_{M}^{2}<<\sigma_{B}^{2}
$$

If the variance of noises are negligible to the variance of $A$ and $B$, the correlation coefficient of $X+A+N$ and $Y+B+M$ will be the same as that of $A$ and $B$.

Taking advantage of this feature, we can add the same signal to both voltage measurements, which means $r_{A, B}$ is 1 . The added signals should be negligible when there are persistent inputs, meanwhile, the variance of the two signals should be larger than that of the noises, such that the correlation coefficient of the two voltages will be close to 1 when the batteries are at rest.

A simple design is to add a square wave with the amplitude of 3 times the standard deviation of the noise standard deviation, namely, 9 times the variance of the noise, as illustrated in 


$$
r_{V_{1}+S W+N, V_{2}+S W+M}=\frac{\operatorname{cov}_{S W, S W}}{\sqrt{\sigma_{S W}^{2}+\sigma_{N}^{2}} \sqrt{\sigma_{S W}^{2}+\sigma_{M}^{2}}}=\frac{9 \sigma_{N}^{2}}{10 \sigma_{N}^{2}}=0.9
$$

where $S W$ is the additive square wave. As a result, the correlation coefficient is close to 0.9 when the batteries are at rest. Clearly, when the amplitude of the square wave is larger, the correlation coefficient is closer to 1 when the battery is at rest. However, the increase in amplitude also decreases the detection sensitivity to the actual voltage drop. In the design of the additive square wave, 0.9 is a reasonable objective given that the threshold is 0.5 in this paper.

The period of the square wave should be smaller than the window size. A trivial selection of period can be 2 samples.

\subsection{Fault isolation}

In real applications, tens or hundreds of cells are connected in series. Here we assume only the minority of the cells may have short circuit fault at the same time. Otherwise, the short circuit fault can be easily detected by module or pack level voltage monitoring.

In order to acquire the status of each battery cell, the correlation coefficients for every pair of neighboring cells need to be calculated, including that for the first and last cell, as illustrated in Fig. 1. When a fault occurs on one of the cells, the two related correlation coefficients drop and the fault location can be isolated by the overlapped index number. For example, when both $r_{V_{1}, V_{2}}$ and $r_{V_{2}, V_{3}}$ demonstrate a sudden drop, it indicates a fault on $V_{2}$ because it is not in the same

trend as those of $V_{1}$ and $V_{3}$. The same strategy can be applied when multiple faults occurs, as long as the fault cells are the minority of the whole pack. 


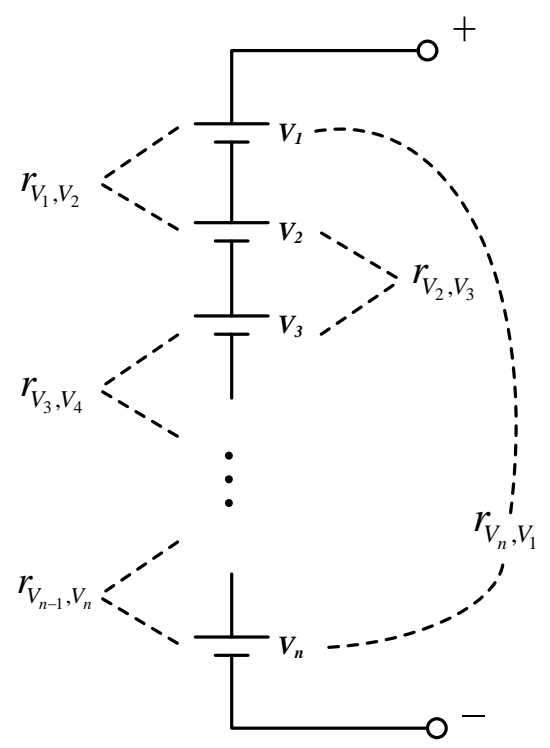

Fig. 1. Correlation coefficient calculation for every pair of neighboring cells.

Table 1 Specification of the batteries under test.

\begin{tabular}{ll}
\hline Battery cell type & Cylindrical 18650 \\
\hline Nominal voltage & $3.2 \mathrm{~V}$ \\
Nominal rated capacity & $1.35 \mathrm{Ah}$ \\
Charge voltage & $3.65 \mathrm{~V}$ \\
Discharge cut-off voltage & $2.5 \mathrm{~V}$ \\
Max pulse discharge & $4.05 \mathrm{~A}$ \\
\hline
\end{tabular}

\section{Simulation}

\subsection{Simulation setup}

The fault conditions are simulated to demonstrate the feasibility of the proposed detection method. First, an experiment is conducted to apply an urban dynamometer driving schedule (UDDS) to two battery cells connecting in series. The specification of the batteries under test is given in Table 1, and the voltage responses of the two cells, $V_{1}$ and $V_{2}$ are given in Fig. 2. The sampling time in the experiment is $0.1 \mathrm{~s}$. 


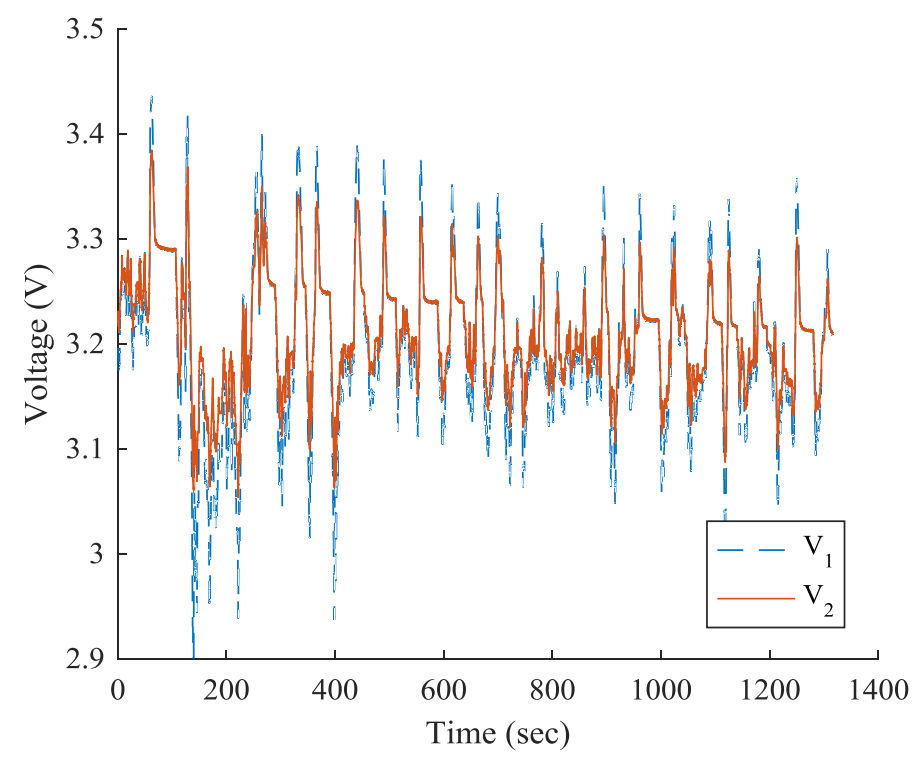

Fig. 2. Voltage responses of two batteries cells used in simulation (without fault signal).

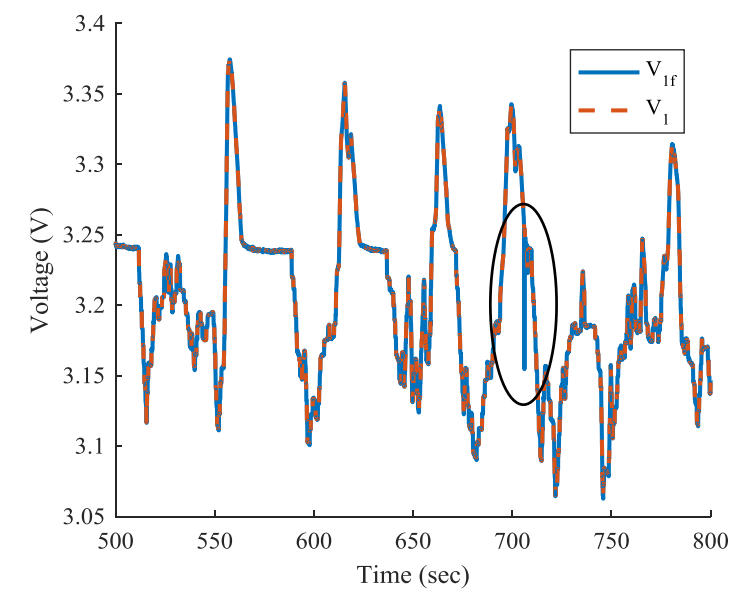

(a) The fault signal added.

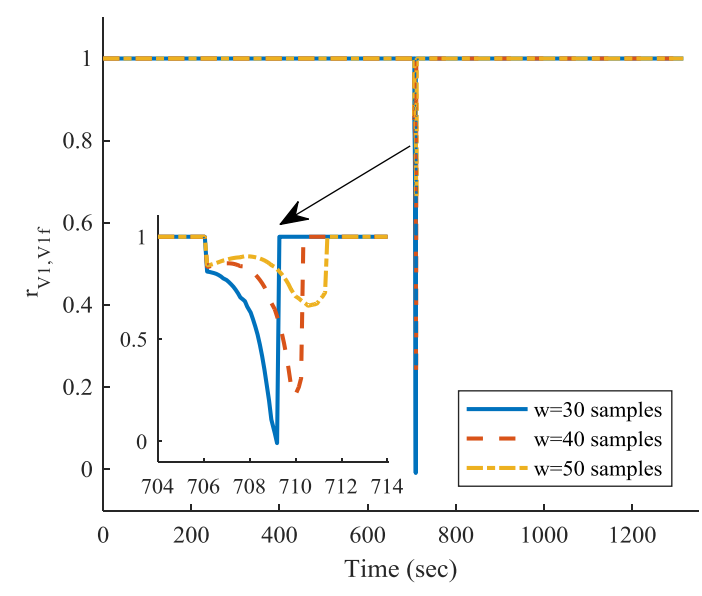

(b) Detection results with inset at faults.

Fig. 3. Demonstration of basic detection principle for short circuits.

Then, a fault signal is constructed by reducing one voltage sample by $100 \mathrm{mV}$ to simulate the sudden voltage drop recovery at the initial phase of internal short circuit. The fault signal is added to $V_{l}$ and denote the synthesized data as $V_{l f}$. In order to demonstrate the basic working principle of the detection algorithm, the correlation coefficient of $V_{l}$ and $V_{l f}$ are first calculated. It needs to be noted that, except the fault signal added, the two voltage responses are exactly the 
same, including the measurement noises. The two voltages are plotted in Fig. 3(a), with the added fault highlighted with an ellipse. The corresponding correlation coefficient of the two voltages are provided in Fig. 3(b) with inset of detection at fault. The moving window sizes are 30, 40 and 50 samples.

After that, independent white noises with the same standard deviation of $1 \mathrm{mV}$ are added to $V_{l}$ and $V_{l f}$, respectively, to emulate the noisy measurements. The noisy voltages, $V_{l, n}$ and $V_{l f, n}$ are given in Fig. 4(a), and the correlation coefficient of them is calculated in Fig. 4(b).

Later, the square wave discussed in Sec. 3.3 is added to $V_{l, n}$ and $V_{l f, n}$, denoting as $V_{l, n, s}$ and $V_{l f, n, s}$, and the corresponding correlation coefficient is plotted in Fig. 4(c). The mean value of the square wave is zero, the amplitude is $3 \mathrm{mV}$ and the period is 2 samples.

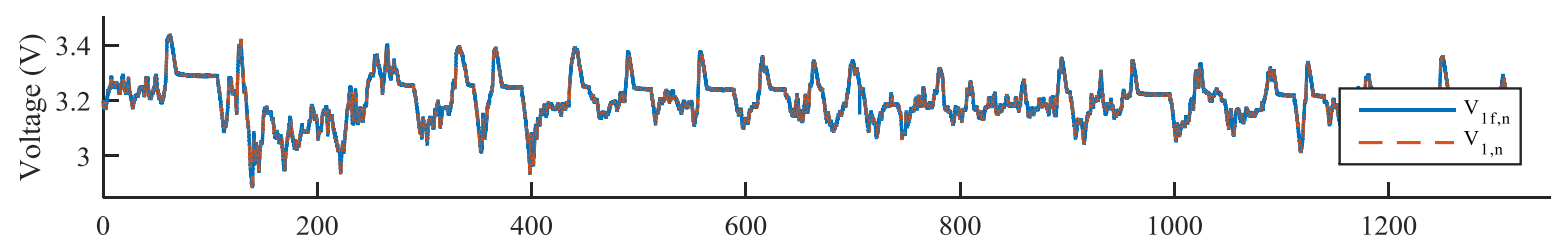

(a) $\mathrm{V}_{1 f, \mathrm{n}}$ and $\mathrm{V}_{1, \mathrm{n}}$

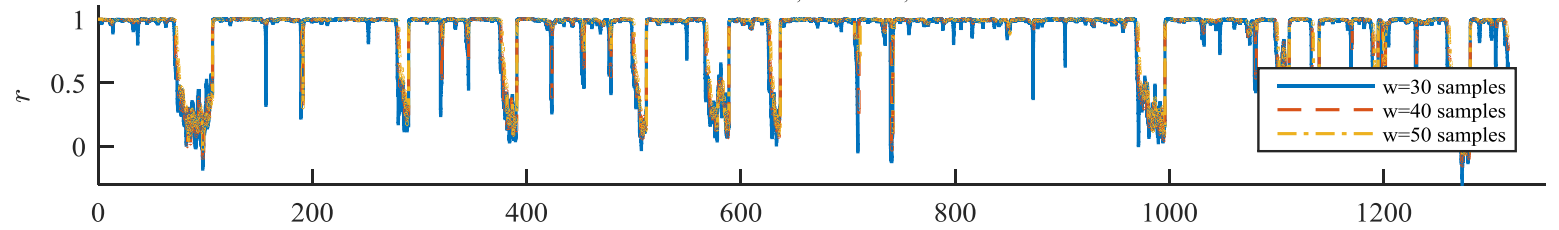

(b) Correlation coefficient with independent noise added

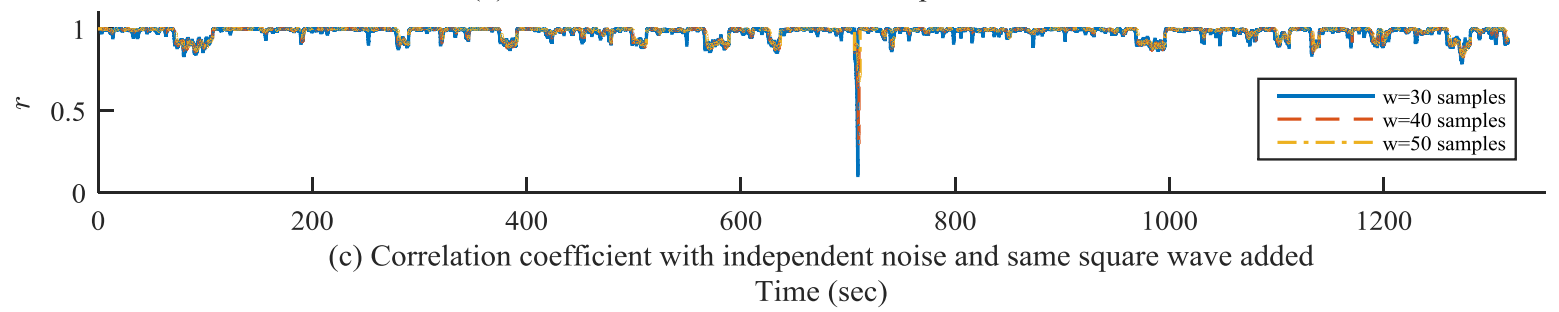

Fig. 4. The drop in $r$ when batteries are at rest is greatly reduced with added square wave.

Finally, real fault detections are simulated by calculating the correlation coefficient of $V_{l f, s}$ and $V_{2, s}$. In this simulation, the window size is 30 samples, and the duration of the voltage drop, 
denoted as $d$, are varied to be 1, 5, 10, 30, 40 and 50 samples, as shown in Fig. 5(a) and Fig. 5(b). A threshold of 0.5 is marked as dashed black lines and is selected to trigger the fault detection.

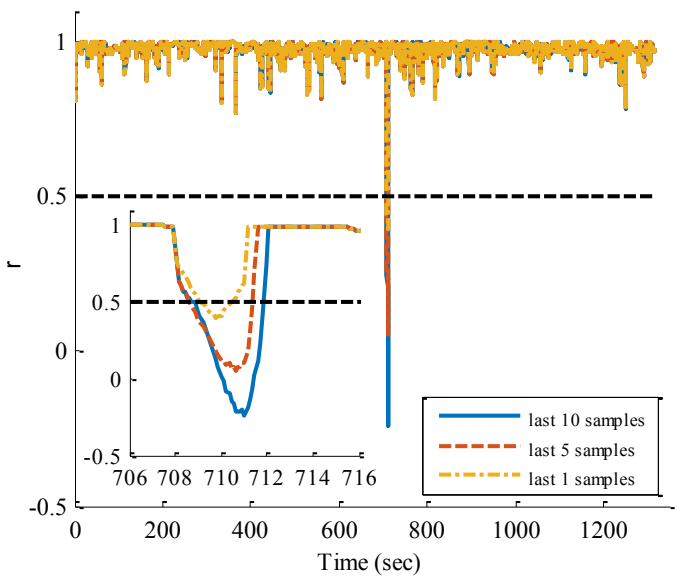

(a) Correlation coefficients when $d<w$.

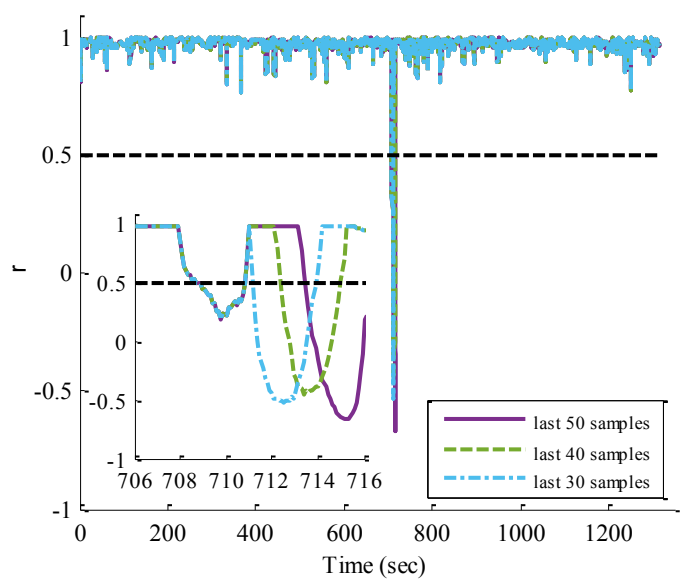

(b) Correlation coefficients when $d \geq w$.

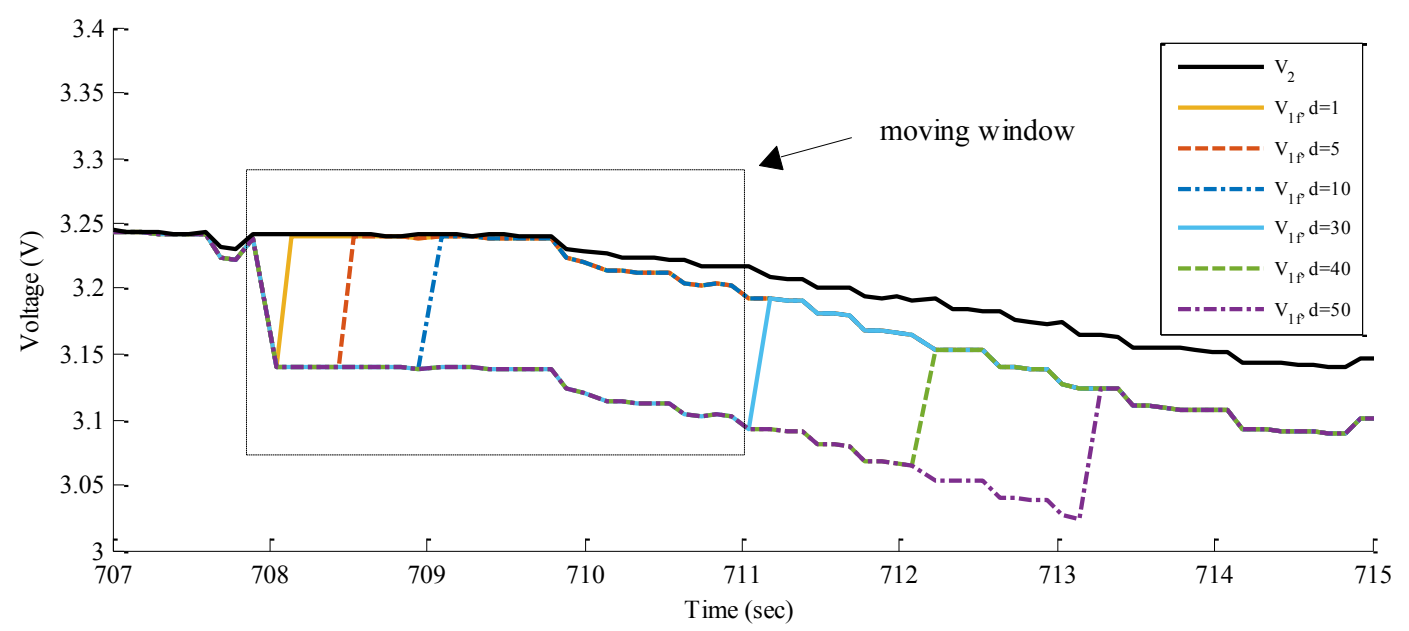

(c) Voltage variations within the moving window.

Fig. 5. The correlation coefficient variation as samples of the voltage drop increases.

\subsection{Discussion of simulation results}

It can be learnt from Fig. 2 that the internal resistances of the two batteries under test are different. Given the same current input, the voltage difference between the two cells can be larger than $100 \mathrm{mV}$ at around $150 \mathrm{~s}$. This large difference will trigger false positive faults if the voltage difference threshold method is applied, or if only one model is tracked online in the 
model based detection method. Fig. 3(a) shows that the fault signal is added to $V_{l}$ at around 700 s. The voltage value at fault is not out of the voltage operation range given in Table 1 , and thus cannot be detected by the voltage threshold method.

The correlation coefficient calculated in Fig. 3(b) manages to capture the off-trend voltage drop recovery, and the fault is flagged by the drop in the correlation coefficients. The comparisons among the calculation with three different window sizes indicate that a smaller window size leads to higher sensitivity to abnormal voltage variations. Meanwhile, when independent white noises are added to the voltage measurements, the correlation coefficient provided in Fig. 4(b) exhibits more fluctuations than that in Fig. 3(b). It needs to be noted that as the size of the moving window increases, part of the fluctuations is smaller, as the ones at around $160 \mathrm{~s}$, owing to the reduced sensitivity to noises.

However, part of the fluctuations remains the same in spite of the variation in window sizes, as the ones at around $100 \mathrm{~s}$. If a closer look is given to the voltages in Fig. 4(a), it can be found that whenever there is a voltage plateau in measurements, there is an unrecoverable fluctuation in the correlation coefficient. Actually, this phenomenon has been well-explained by Sec. 3.3 that when the batteries are at rest, the correlation coefficient will drop as indicated in (7). The proposed solution is to add a square wave to the voltage measurements with an amplitude of 3 $\mathrm{mV}$ and a period of 2 samples. Fig. 4(c) presents the correlation coefficient with the square wave added, indicating that the induced fault can be easily identified.

In the simulation, the induced fault is detected with the proposed correlation based method, whereas the other detection methods lead to various issues. The detection results are summarized in Table 2. 
Table 2 Comparison of the simulated short circuit detection results.

\begin{tabular}{lcc}
\hline Detection method & True fault & False fault \\
\hline Voltage threshold based & & \\
Voltage difference threshold based & $\checkmark$ & $\checkmark$ \\
Model based & $\checkmark$ & $\checkmark$ \\
Correlation based & $\checkmark$ &
\end{tabular}

It is understood that the correlation coefficient measures the similarity of the two signals. It can be inferred when the voltage drop led by faults lasts longer within the moving window, the similarity of the two voltage measurements degrades further, and thus the drop in the correlation coefficient will be larger, as illustrated in Fig. 5(a). This ensures the robustness of this detection method when multiple samples in the voltage drop are captured.

The simulated fault voltages $\mathrm{V}_{1 \mathrm{f}}$ with different lengths are demonstrated in Fig. 5(c). The different faults start at the same time, but recover at different time instants. It can be observed that when the voltage drop lasts longer than the length of the moving window, the batch-wise voltage data within the moving window are the same at the initial stage of the short circuits, as the cases of 30, 40 and 50 samples. Hence, in these cases, the variations in the correlation coefficients are the same at the initial stages, regardless of the length of the voltage drop, as shown in Fig. 5(b). Therefore, the fault detection times for short circuits with long durations are the same, as summarized in Table 3. This property makes the proposed method applicable to external short circuit detection as well, where the voltage drop may be longer than the moving time window.

Table 3 Fault detection times of correlation based method for different short circuit durations.

\begin{tabular}{ccccccc}
\hline Fault duration (sample) & 1 & 5 & 10 & 30 & 40 & 50 \\
\hline Detection time (sec) & 1.1 & 0.7 & 0.7 & 0.7 & 0.7 & 0.7 \\
\hline
\end{tabular}




\section{Experiment}

\subsection{Experiment setup}

An experiment is set up to validate the proposed fault detection approach. The schematics for experimental setup is shown in Fig. 6(a). Four battery cells, same as those introduced in Table 1, are connected in series. A customized BMS is equipped to monitor the voltage, current and temperature of the battery string. All the data are collected with dSPACE Micro-Autobox and saved through ControlDesk in the host PC. The charge/discharge commands are executed by the direct current power source and electronic load connecting in parallel with the battery string. The hardware implementation is provided in Fig. 6(b).

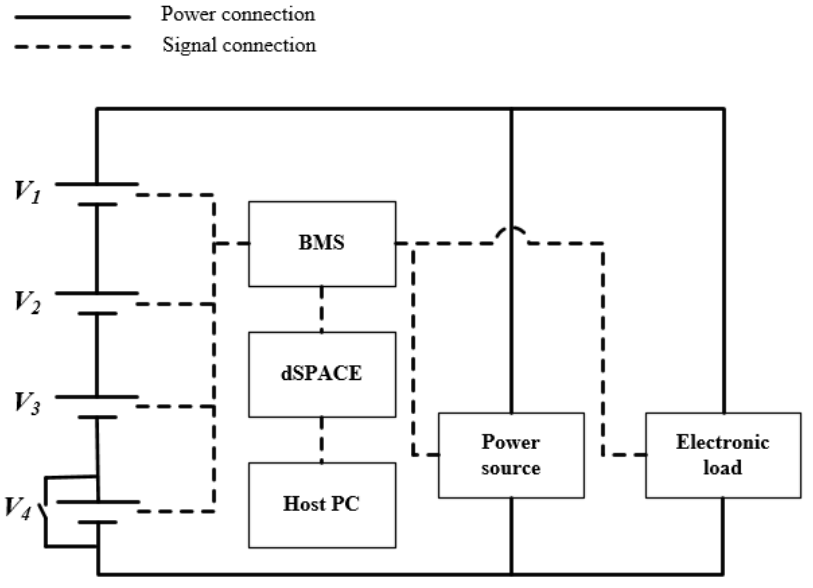

(a) Schematics for experiment setup.

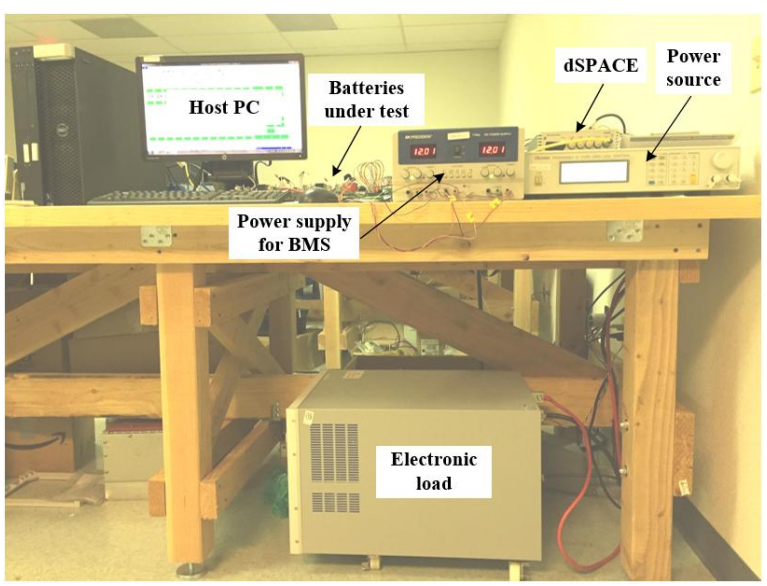

(b) Hardware setup for experiment.

Fig. 6. Experimental setup for validation.

The battery string is then applied with a UDDS cycle in the room temperature. At $42.4 \mathrm{~s}$ of the UDDS cycle, a jump wire with $0.36 \Omega$ resistance is used to short the positive and negative terminal of cell \#4 for $1.3 \mathrm{~s}$. The corresponding voltage responses for the four cells are given in Fig. 7(a), and the temperature responses are given in Fig. 7(b). The sampling interval in the experiment is $0.1 \mathrm{~s}$. 


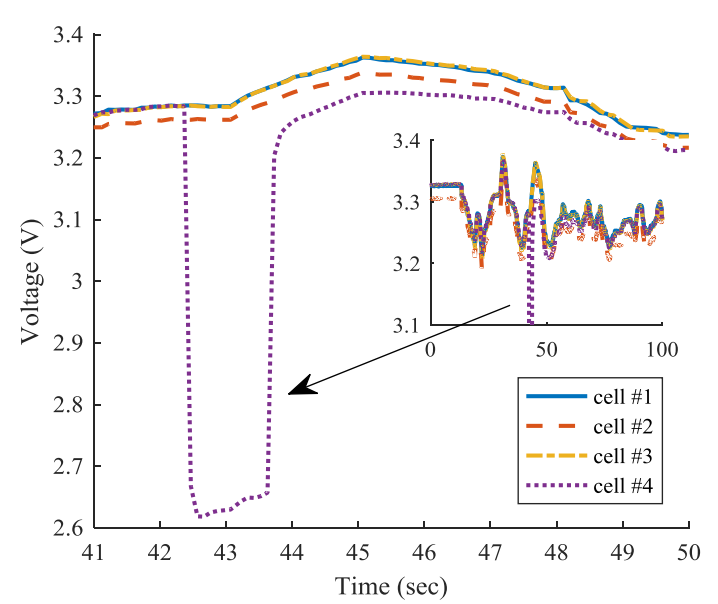

(a) Cell voltages in the experiment.

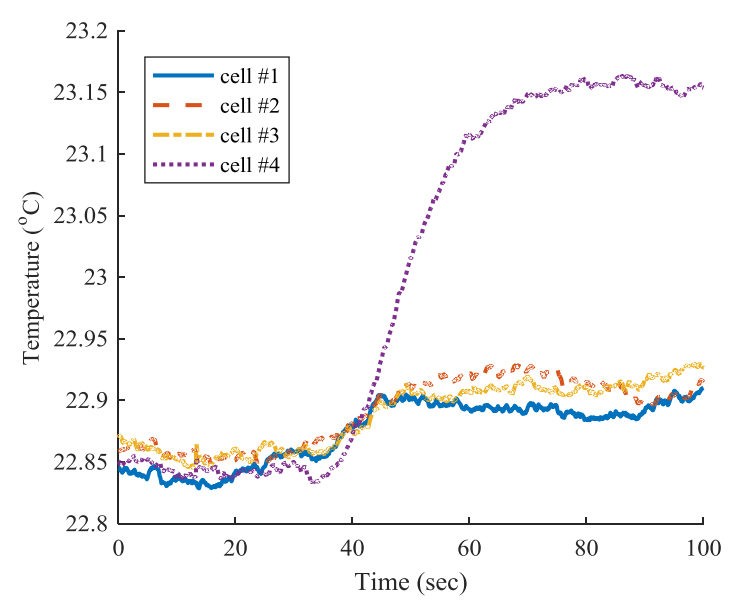

(b) Cell temperatures in the experiment.

Fig. 7. Experiment results.

\subsection{Discussion of experiment results}

It can be observed from Fig. 7(a) that when cell \#4 is shorted by the jump wire, the voltage suddenly drops to approximate $2.6 \mathrm{~V}$, and the voltage recovers after the short is removed. The voltage drop does not touch the discharge voltage limit given in Table 2. From the temperature plot in Fig. 7(b), the temperature response of cell \#4 does have a higher rise than other cells after the fault occurrence, however, the amount is only $0.3{ }^{\circ} \mathrm{C}$, which is negligible to notice in real applications. Since the short circuit current does not pass the current sensor on the BMS board, the short circuit is unobservable from current measurements. Thus the voltage, current or temperature threshold based detection methods do not flag any fault in the scenario.

It is also worth noting that the SoCs of the four batteries under test are different. The OCV of cell \#2 is lower than the average OCV of the other three cells by $22 \mathrm{mV}$. This difference may lead to false detection if the voltage difference threshold method is applied. When the model based method is applied to track the voltage of only one of the cells, it will also result in a false fault detection. 
The correlation coefficients for the neighboring cells are calculated and plotted in Fig. 8. The size of the moving window is 30 samples. The amplitude of the square wave added is $3 \mathrm{mV}$ and the period is 2 samples. The correlation coefficients for the first two neighboring cell pairs are close to 1 in the experiment, indicating these three cells follow the same variation in the whole process. Whereas, the correlation coefficient of cell \#3 and cell \#4 drops abruptly when the fault occurs because of the off-trend voltage drop. The location of the fault can be determined as cell \#4 because the same drop is captured in both $r_{(3,4)}$ and $r_{(4,1)}$.

A threshold value of 0.5 is marked in Fig. 8 to flag the short circuit fault. The voltage drop is captured in the voltage reading at $42.5 \mathrm{~s}$, and the correlation coefficient flags the fault at 42.5 s. It is because the voltage variation is much larger than the normal voltage fluctuations, leading to a large drop in the correlation coefficient once the voltage drop is captured. This demonstrates the prompt response of the proposed fault detection method in real applications.
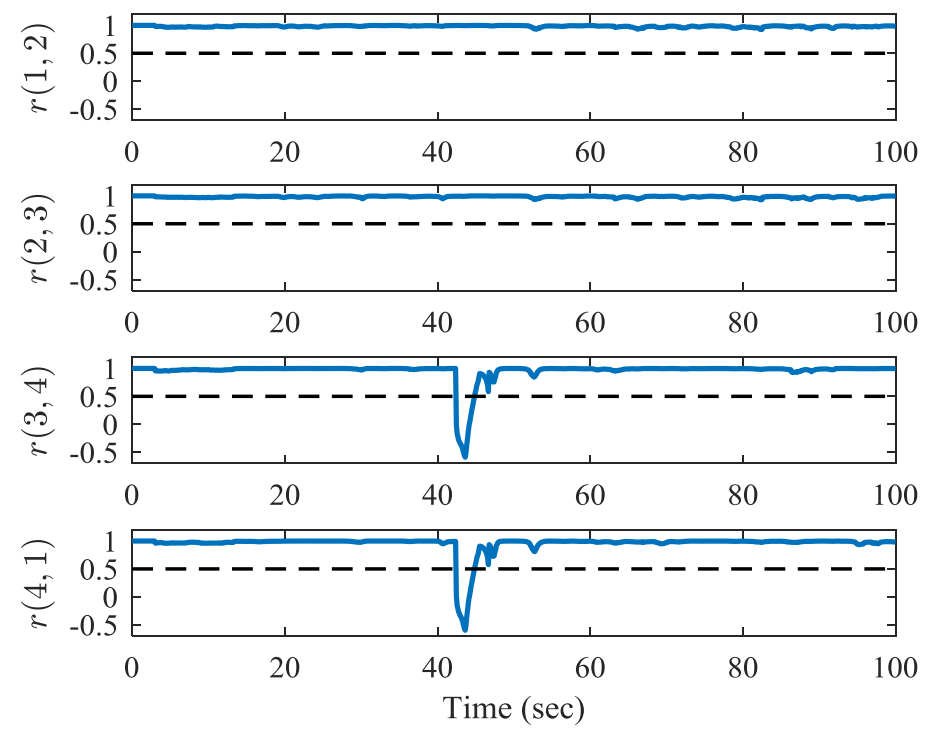

Fig. 8. Correlation coefficients calculated for the neighboring cells.

\section{Further discussion}

\subsection{Key assumptions}


The proposed fault detection method utilizes the measurement of similarity from the correlation coefficient, and determines a fault when the similarity is low. This section discusses the key assumptions which may be violated in real applications and the corresponding mitigation methods.

\subsubsection{White noises}

In the derivation in Sec. 3.3, the noises in the measurement are assumed to be white noises, which is not true in real applications. Except that, given a small number of samples, the noises can hardly exhibit its statistical property under the law of large numbers [38]. As a result, the variance of the noises may be higher, and this can be compensated by adding a square wave with higher amplitudes.

\subsubsection{Synchronized measurement}

The voltages in a vehicular battery pack are usually measured sequentially to save hardware cost. For the experiment in this manuscript, the voltages are sequentially updated, but they are shifted to align with one another in the time domain before the correlation coefficient calculation. Otherwise, one voltage always leads or lags others, leading to false detections. One solution is to shift the measurements in the moving window, and calculate the correlation coefficient afterwards, as done in this manuscript. The other solution is to utilize the crosscorrelation $[39,40]$ which calculates the correlation coefficients of the time shifted version of two signals. The time difference in the cross-correlation calculation will be the sampling interval.

\subsubsection{Minority in fault}

It is discussed in Sec. 3.4 that the fault isolation is not accurate when the majority of the cells is in fault at the same time. In the extreme condition, when the whole pack is in the external short circuit condition, the correlation coefficients are close to 1, because the voltage trends are 
the same. However, when multiple external short circuits occur at the same time, the faults can be easily detected by module or pack level voltage monitoring. In addition, the internal short circuits do not occurs simultaneously in real applications.

\subsubsection{Short with low resistances}

The proposed method captures the abrupt voltage drop by calculating correlation coefficient within a moving window. A thorough study of internal short circuits with different resistances has been conducted in [33]. It shows that not every internal short circuit has abrupt voltage drop, or low resistance short. With large short resistances, the voltage curves follow the same trend over the relatively short moving time windows and thus the fault cannot be identified. Nevertheless, the proposed method is still meaningful because the internal shorts with low resistances require immediate mitigation, and are more dangerous due to the instant excess heat generation. Indeed, the large resistance internal short circuit can also be detected by the correlation based method, when the moving window filter is modified as

$$
W_{i}=\left\{\begin{array}{cc}
1, & i=n-j k, j=0,1, \cdots, w-1 \\
0, & \text { otherwise }
\end{array}\right.
$$

where $W$ is the function of moving window filter, and $k$ is the number that adjusts the time span of the moving window filter. This filter evenly samples $w$ points within a time span of $k w$. It needs to be noted that the moving average filter used in the previous sections is a special case of (11) with $k=1$. When $k$ is large, this filter can capture the voltage behavior over long periods of time, thereby identifying internal shorts with large resistances.

\subsection{Comparison of different detection methods}

Because of the high cost of hardware redundancy, the software redundancy is the most applied fault detection methods in battery systems. The voltage threshold method is the simplest model, which only considers the safe operation range of the system, and is ignorant of the input 
information. It is easy in implementation, but the weakness is that the out-of-range voltage is not a necessary condition for faults. In other words, a battery can still be faulty when the voltage is in the safe range.

An improvement can be made by taking the input into consideration, and then it gives rise to the model based fault diagnostic methods. The methods are able to distinguish the fault conditions when the voltages are within the safe range, but the tradeoff is their substantial effort in maintaining the robustness and accuracy of the battery models in difference situations. Except that, there may be a false positive alarm when a fault is flagged, due to an inaccurate battery model or the inconsistencies among individual cells.

The proposed method directly compares the outputs of the multiple cells, and identifies the fault by the off-trend voltage behavior. The battery model is then not needed, which saves the effort in modeling, because every other cell can be the hardware redundancy of the current cell. It is interesting to note that the voltage difference threshold method is a special case of the proposed method, where it assumes all the cells are the same. This assumption is not true when the SoC and $\mathrm{SoH}$ of the cells varies, and is compensated well by the properties of correlation coefficients. A brief summary of the comparisons of different detection methods is provided in Table 4.

Table 4 Comparison of different detection methods.

\begin{tabular}{lll}
\hline Detection method & \multicolumn{1}{c}{ Advantages } & Disadvantages \\
\hline Voltage threshold & Easy implementation. & False negative faults within the \\
Model based & Fault detection in the operation & Substantial modeling work; \\
& range. & ambiguity in fault detection. \\
Voltage difference threshold & No modeling work. & False positive faults when SoCs \\
\hline
\end{tabular}


or SoHs are not consistent.

Correlation based

No modeling work;

Need to be combined with

Fault detection with inconsistent module/pack level monitoring.

SoCs or SoHs.

\section{Conclusion}

A correlation based fault detection method is proposed in this manuscript, which does not require hardware and analytical redundancy, thus saving the hardware cost and effort in system modeling.

The concept of correlation coefficient is first introduced. It is proved that the correlation coefficient can detect the initial stage of short circuits by capturing the off-trend voltage drop, and reflect the variation to the drop in correlation coefficient, in spite of the cell inconsistencies in $\mathrm{SoC}$ or $\mathrm{SoH}$.

Next, the correlation coefficient is expressed in the recursive form for online application. A moving average window is applied to keep the most recent voltage trends of the cells, while maintaining the detection sensitivity to short circuit faults. A square wave is added to the voltage measurements to prevent the false detection when the batteries are at rest. In addition, it is analyzed that the short circuit fault can be isolated by identifying the overlapped cell in the dropped correlation coefficients.

Moreover, simulation and experiment results validated the analysis, and demonstrated the scenarios where the proposed method can robustly identify the faults, while the voltage threshold, voltage difference threshold and model based method lead to either false negative faults or false positive faults.

At last, the key assumptions in the proposed method are discussed. It is explained that the proposed method can detect the short circuits with a large short resistance by modification of the 
moving window. The comparison with other detection methods shows that the proposed method does not require modeling work, and provides robust short circuit detection regardless of the inconsistencies within the battery pack.

The future work of this study is to enhance the proposed detection method by adding the large resistance short circuit detection.

\section{Acknowledgements}

The authors would like to acknowledge the funding support from Nanjing Golden Dragon Bus Co., Ltd., and the U.S. Department of Energy under the Graduate Automotive Technology Education Center program.

\section{Reference}

[1] X. Zhang, C. Mi, Vehicle Power Management: Modeling, Control and Optimization, Springer Science \& Business Media, 2011.

[2] N. Williard, W. He, C. Hendricks, M. Pecht, Energies, 6 (2013) 4682-4695.

[3] B. Smith, US Department of Transportation, National Highway Traffic Safety Administration, (2012) 1.

[4] Q. Wang, P. Ping, X. Zhao, G. Chu, J. Sun, C. Chen, Journal of power sources, 208 (2012) 210-224.

[5] L. Lu, X. Han, J. Li, J. Hua, M. Ouyang, Journal of Power Sources, 226 (2013) 272-288.

[6] C. Weng, X. Feng, J. Sun, H. Peng, Applied Energy, 180 (2016) 360-368.

[7] R. Xiong, F. Sun, Z. Chen, H. He, Applied Energy, 113 (2014) 463-476.

[8] Z. Chen, B. Xia, C.C. Mi, R. Xiong, Industry Applications, IEEE Transactions on, 51 (2015) 4121-4129.

[9] Y. Shang, C. Zhang, N. Cui, J.M. Guerrero, IEEE Transactions on Power Electronics, 30 (2015) 3731-3747.

[10] X. Feng, J. Sun, M. Ouyang, X. He, L. Lu, X. Han, M. Fang, H. Peng, Journal of Power Sources, 272 (2014) 457-467.

[11] B. Xia, C. Mi, Journal of Power Sources, 308 (2016) 83-96.

[12] B. Xia, C. Mi, Z. Chen, B. Robert, in: Transportation Electrification Conference and Expo (ITEC), 2015 IEEE, IEEE, 2015, pp. 1-7.

[13] X. Feng, J. Sun, M. Ouyang, F. Wang, X. He, L. Lu, H. Peng, Journal of Power Sources, 275 (2015) 261 -273. 
[14] C.-S. Kim, J.-S. Yoo, K.-M. Jeong, K. Kim, C.-W. Yi, Journal of Power Sources, 289 (2015) 41-49.

[15] R. Angerbauer, A. Reitzle, J. Fetzer, in, Google Patents, 2014.

[16] B. Xia, Z. Chen, C. Mi, B. Robert, in: Transportation Electrification Conference and Expo (ITEC), 2014 IEEE, IEEE, 2014, pp. 1-7.

[17] W. Cai, H. Wang, H. Maleki, J. Howard, E. Lara-Curzio, Journal of Power Sources, 196 (2011) 7779-7783.

[18] J. Asakura, T. Nakashima, T. Nakatsuji, M. Fujikawa, in, Google Patents, 2012.

[19] Y. Barsukov, J. Qian, Battery power management for portable devices, Artech house, 2013.

[20] E. Sahraei, J. Campbell, T. Wierzbicki, Journal of Power Sources, 220 (2012) 360-372.

[21] L. Greve, C. Fehrenbach, Journal of Power Sources, 214 (2012) 377-385.

[22] W. Fang, P. Ramadass, Z.J. Zhang, Journal of Power Sources, 248 (2014) 1090-1098.

[23] H. Wang, S. Simunovic, H. Maleki, J.N. Howard, J.A. Hallmark, Journal of Power Sources, 306 (2016) 424430.

[24] H. Maleki, J.N. Howard, Journal of Power Sources, 191 (2009) 568-574.

[25] T. Hatchard, S. Trussler, J. Dahn, Journal of Power Sources, 247 (2014) 821-823.

[26] C.J. Orendorff, E.P. Roth, G. Nagasubramanian, Journal of Power Sources, 196 (2011) 6554-6558.

[27] S. Santhanagopalan, P. Ramadass, J.Z. Zhang, Journal of Power Sources, 194 (2009) 550-557.

[28] X. Feng, M. Fang, X. He, M. Ouyang, L. Lu, H. Wang, M. Zhang, Journal of Power Sources, 255 (2014) 294301.

[29] G.-H. Kim, A. Pesaran, R. Spotnitz, Journal of Power Sources, 170 (2007) 476-489.

[30] C. Zhang, S. Santhanagopalan, M.A. Sprague, A.A. Pesaran, Journal of Power Sources, 290 (2015) 102-113.

[31] K.-C. Chiu, C.-H. Lin, S.-F. Yeh, Y.-H. Lin, K.-C. Chen, Journal of Power Sources, 251 (2014) 254-263.

[32] A. Singh, A. Izadian, S. Anwar, Journal of Power Sources, 268 (2014) 459-468.

[33] X. Feng, C. Weng, M. Ouyang, J. Sun, Applied Energy, 161 (2016) 168-180.

[34] B. Xia, X. Zhao, R. de Callafon, H. Garnier, T. Nguyen, C. Mi, Applied Energy, 179 (2016) 426-436.

[35] G.-H. Kim, K. Smith, J. Ireland, A. Pesaran, Journal of Power Sources, 210 (2012) 243-253.

[36] B. Xia, T. Nguyen, J. Yang, C. Mi, Journal of Power Sources, 334 (2016) 12-22.

[37] M.J. Campbell, T.D.V. Swinscow, Statistics at square one, John Wiley \& Sons, 2011.

[38] K. Yao, J. Gao, IEEE Transactions on Fuzzy Systems, 24 (2016) 615-621. 
[39] J. Gao, Q. Cheng, X. Wang, F. Tan, Y. Zhang, D. Yu, in: Electricity Distribution (CICED), 2016 China International Conference on, IEEE, 2016, pp. 1-5.

[40] T.J. Rato, M.S. Reis, Chemometrics and Intelligent Laboratory Systems, 125 (2013) 101-108. 